\title{
Scrub typhus in pregnancy presenting with permanent hearing loss: A case report
}

\author{
Sangay Tshering ${ }^{1}$, Namkha Dorji ${ }^{2}$, Dago $\mathrm{Dem}^{3}$, and Tandin $\mathrm{Om}^{3}$ \\ ${ }^{1}$ JDWNR Hospital \\ ${ }^{2}$ Jigme Dorji Wangchuck National Referral Hospital \\ ${ }^{3}$ Faculty of Post Graduate Medicine, Khesar Gyalpo University of Medical Sciences of \\ Bhutan
}

March 26, 2021

\begin{abstract}
Introduction: Scrub typhus can present with audiological symptoms. Case: A pregnant mother presented with persistent fever, pneumonia and hearing loss. Investigation showed positive serology for scrub typhus. Conclusion: Clinicians must be aware of audiological presentations in scrub typhus. Possibly, permanent hearing loss was due to meningoencephalitis and hormone induced immunomodulation
\end{abstract}

Scrub typhus in pregnancy presenting with permanent hearing loss: A case report

Running title: scrub typhus and hearing loss

Abstract

Introduction: Scrub typhus can present with audiological symptoms.

Case: A pregnant mother presented with persistent fever, pneumonia and hearing loss. Investigation showed positive serology for scrub typhus.

Conclusion: Clinicians must be aware of audiological presentations in scrub typhus. Possibly, permanent hearing loss was due to meningoencephalitis and hormone induced immunomodulation.

Keywords: hearing loss; pregnancy; scrub typhus.

Introduction

Scrub typhus is a mite borne infectious disease caused by Orientia tsutsugamushi. The vector and reservoir for this disease is the larval trombiculid mites also known as chiggers. The southern belt of Bhutan is seasonally endemic to scrub typhus with highest occurrence in farmers. ${ }^{1}$

The clincal manifestations of this non specific febrile illness are intense headache and myalgia. Some patients develop generalized lymphadenopathy, macular or maculopapular non pruritic rashes and an eschar. Rare and atypical presentations such as acute reversible auditory symptoms like hearing loss, tinnitus and otalgia have been reported. ${ }^{2,3}$ Possible mechanism for such presentations are immune mediated vasculitis leading to cochlear neuroinflammation resulting from exaggerated Th1 cellular immune response. ${ }^{3,4}$ The severity of infection can vary from mild symptoms to severe multiorgan failure. Mortality rates were higher in those developing pneumonia, delirium, myocarditis and elderly population. ${ }^{5,6}$ 
The clinical diagnosis accuracy remains non specific due to largely overlapping symptoms seen in other tropical infections like dengue, malaria and leptospirosis. Most patients develop thrombocytopenia, elevated hepatic enzymes and deranged renal functions. Leucopenia or leucocytosis may develop but most patients present normal total leucocyte count. ${ }^{7}$

Since the audiological symptoms related to scrub typhus discussed in the literature were transient or reversible, we hereby present this case with permanent hearing loss in a pregnant woman following scrub typhus infection.

Case presentation

A previously healthy 39 year old G6P5 farmer at 34 weeks gestation was referred from Punakha District Hospital with 5 days history of fever, headache, generalized bodyache, shortness of breath and cough. There were no audiological, gastrointestinal or urinary symptoms. On admission to the maternity ward, she was ill looking but conscious with tachypnea, high grade fever and pallor. There was no cervical lymphadenopathy, icterus, body rashes, eschar or ear infection. Her respiratory rate was $26-30 /$ min, pulse rate of 120 beats per minute and blood pressure of 110/70 mm Hg. Cardiac auscultation revealed ejection systolic murmur at left lower sternal edge which was probably due to hyperdynamic circulation in anemia.. There was coarse crepitation noted in bilateral lung fields. Abdominal examination revealed no hepatosplenomegaly. Cardiotocograph was reassuring.

Presumptive diagnosis of community acquired pneumonia was made and empirical treatment started with intravenous ceftriaxone and oral erythromycin. Antenatal corticosteroid was administered as per the hospital protocol. One unit of packed red cell was transfused.

Laboratory investigation reports showed moderate anemia (Hb 7.9gm\%), raised C-reactive protein $(19.9 \mathrm{mg} / \mathrm{l})$, and mild transaminitis. Renal function was normal. Sputum culture showed Klebsiella pneumoniaewhich was sensitive to Ciprofloxacin and resistant to Ceftriaxone. Blood and urine culture were sterile. Dengue serology and malaria parasite smear were negative. Chest X ray (Figure 1) showed features suggestive of pneumonia. Echocardiogram was done to rule out cardiac causes of febrile illness and the findings were normal. 


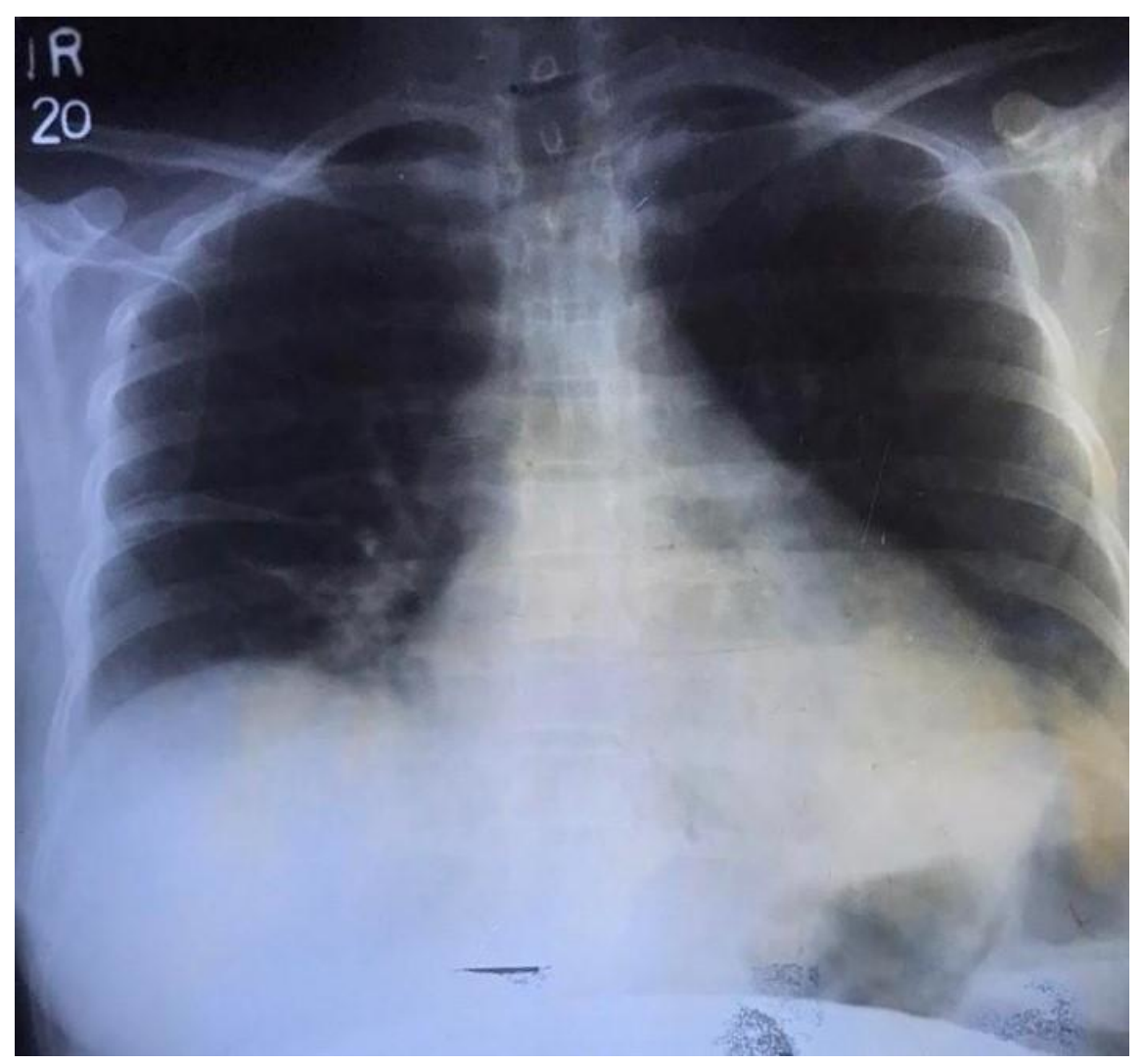

Figure 1: CXR postero anterior view showing segmental consolidation of right lower zone

There was no clinical improvement after 72 hours of antibiotic therapy. Intravenous ciprofloxacin was administered based on sputum culture and antibiotic sensitivity test for 3 days. Her respiratory symptoms improved but still remained febrile. She complained of bilateral hearing loss after 3-4 days of admission and appeared delirious on examination. She didnot complain of tinnitus or otalgia. Urgent MRI brain showed no significant abnormalities although minor details could not be reported due to frequent motion by the agitated patient. Repeat serological investigation showed positive IgM for scrub typhus. Other hematological investigations including complete blood count, liver functions and renal functions did not show significant changes compared to previous results. She responded to oral azithromycin 1 gm daily for 03 days. Induction of labour was done in view of fetal jeopardy and delivered a male baby weighing 2100 grams with Apgar score of 9 and 10 at 1 and 5 minutes respectively. There were no intrapartum and immediate post partum complications. Audiology examination done postpartum 3rd day showed profound bilateral hearing loss (Figure 2). Audiology re-assessment was done at 6 and 12 weeks postpartum.

Clinically, there was no improvement in her hearing and repeat Pure Tone Audiometry (PTA) at 12 weeks (Figure 3) still showed profound bilateral hearing loss similar to the initial assessment. A retrospective diagnosis of permanent hearing loss following scrub typhus in pregnancy was made. Auditory Brainstem Response (ABR) was not performed as MRI brain performed earlier did not identify any retrocochlear lesions and PTA findings were sufficient to assess the hearing threshold. She was advised to adopt total communication as an alternative. 


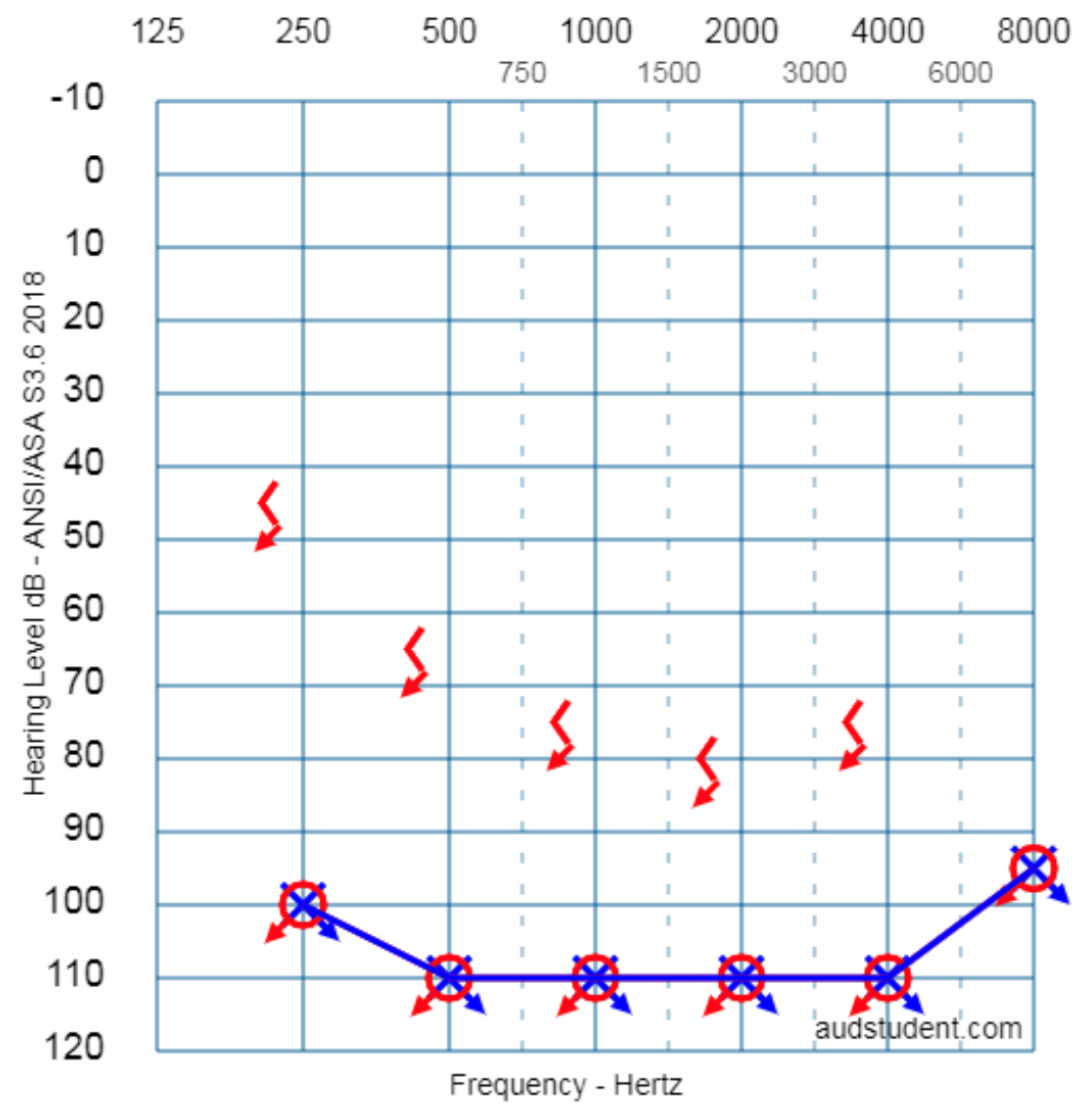

Figure 2: Initial Pure Tone Audiometry 


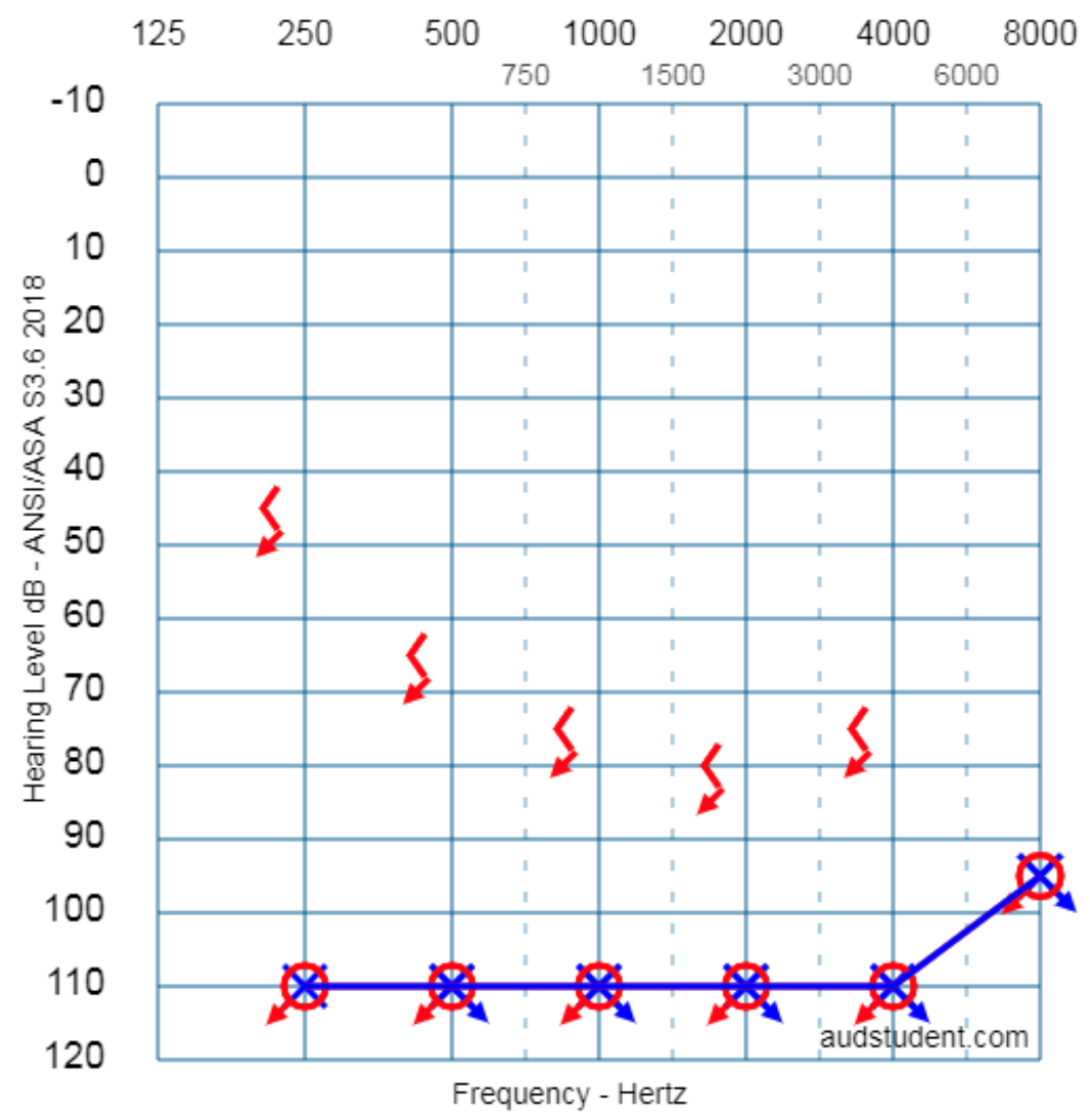

Figure 3: Repeat Pure Tone Audiometry at 12 weeks postpartum

Discussion

This is a case of scrub typhus in pregnancy with respiratory and possible meningoencephalitis complications. The diagnosis of scrub typhus in our case was delayed due to predominant respiratory symptoms, absence of eschar and the initial false negative serology result with SD Bioline Tsutsugamushi RDT (Standard Diagnostics Inc, Korea). ${ }^{8}$ The appearance of audiological symptoms such as hearing loss in acute undifferentiated febrile illness should raise suspicion for scrub typhus infection. ${ }^{2}$ Audiological symptoms were present in almost $30 \%$ of the cases. ${ }^{2}$ A hospital based review of records of 33 pregnant women with scrub typhus infection reported $4.5 \%$ incidence although none of the cases reported hearing loss. ${ }^{9}$ Similar to non pregnant patients, the severity of disease is associated with delayed diagnosis and treatment leading to multiorgan failure. It is associated with poor obstetric outcome such as maternal admission to intensive care unit, miscarriage, fetal demise and preterm birth especially in $3^{\text {rd }}$ trimester in atleast $50 \%$ of the cases. ${ }^{9,10}$ Consistent with other studies, maternal, fetal and neonatal outcomes were favorable in our case as there was no multi organ dysfunction. There are also few case reports on vertical transmission. ${ }^{11}$ However the baby's serology in our case came negative for scrub typhus infection. Case series reported by Premaratna et al have shown objective improvement in hearing loss in those who recovered without any complications. ${ }^{2}$ All cases except one responded to course of tetracycline/chloramphenicol. One patient died due to myocarditis and meningoencephalitis due to delayed diagnosis. As a result of delayed diagnosis, our case developed clinical features suggestive of meningoencephalitis. The permanent loss of hearing might have been a sequela to this complication. A hypothetic explaination on rarity of literature on permanent hearing loss due to scrub typhus in pregnancy may be due to shift in Th 1 to Th 2 immune modulation. ${ }^{12}$ Possibly, neuroinflammation may be less pronounced due to the Th1 to Th2 shift in early stage of infection followed by overwhelming 
infection at later stage leading to more generalized meningoencephalitis. A reasonable postpartum time period of 3 months was allowed to recover from pregnancy associated hearing loss as seen in some healthy women. ${ }^{13}$ Telephonic follow up with spouse at 6 months revealed no subjective improvement in hearing.

This case report is probably the first of its kind in describing the permanent hearing loss following scrub typhus in pregnancy. Although no cause effect relationship can be deduced from this report, we still generate a hypothesis based on hormonal mediated immunomodulation and permanent hearing loss in scrub typhus infection.

Conclusion

As clinicians, we need to be vigilant about these rare and atypical presentations given the endemic nature of scrub typhus in southern belt of the country. Timely diagnosis and appropriate treatment is the key aspect to prevent further complications. Possibly, hearing loss in pregnancy due to scrub typhus could be permanent due to synergistic effect of hormone induced changes in immunomodulation and meningoencephalitis.

Acknowledgement: We would like to thank Mr. Sangay Tshering, Audiologist at the JDWNRH, Thimphu for the providing the results of Pure Tone Audiometry in digital form.

Conflict of interest: There is no conflict of interest in publication of this article.

Funding: None.

Ethical approval: Ethical approval is not needed for case report in de-identified patients.

List of abbreviations

PTA: Pure Tone Audiometry

ABR: Auditory Brainstem Response

Authors contribution

ST was involved in conception and design, acquisition of data, analysis and interpretation of data, revising it critically for important intellectual content, final approval of the version to be published and agreed to be accountable for all aspects of the work.

ND was involved in conception and design, acquisition of data, revising it critically for important intellectual content, final approval of the version to be published and agreed to be accountable for all aspects of the work.

DD was involved in conception and design, acquisition of data, interpretation of data, revising it critically for important intellectual content, final approval of the version to be published and agreed to be accountable for all aspects of the work.

TO was involved in conception and design, acquisition of data, interpretation of data, revising it critically for important intellectual content, final approval of the version to be published and agreed to be accountable for all aspects of the work.

\section{References}

1. Dorji K, Phuentshok Y, Zangpo T, Dorjee S, Dorjee C, Jolly P, et al. Clinical and Epidemiological Patterns of Scrub Typhus, an Emerging Disease in Bhutan. Trop Med Infect Dis. 2019 Mar;4(2).

2. Premaratna R, Chandrasena TG, Dassayake AS, Loftis AD, Dasch GA, de Silva HJ. Acute hearing loss due to scrub typhus: a forgotten complication of a reemerging disease. Clin Infect Dis. 2006;42(1):6-8.

3. Kang JI, Kim DM, Lee J. Acute sensorineural hearing loss and severe otalgia due to scrub typhus. BMC Infect Dis. 2009;9:173. 
4. Soong L. Dysregulated Th1 Immune and Vascular Responses in Scrub Typhus Pathogenesis. J Immunol. 2018;200(4):1233-40.

5. Peter JV, Sudarsan TI, Prakash JAJ, Varghese GM. Severe scrub typhus infection: Clinical features, diagnostic challenges and management. World J Crit care Med. 2015 Aug;4(3):244-50.

6. Taylor AJ, Paris DH, Newton PN. A Systematic Review of Mortality from Untreated Scrub Typhus (Orientia tsutsugamushi). PLoS Negl Trop Dis. 2015;9(8):e0003971.

7. Kim D-M, Kim SW, Choi S-H, Yun NR. Clinical and laboratory findings associated with severe scrub typhus. BMC Infect Dis. 2010 Apr;10:108.

8. Pote K, Narang R, Deshmukh P. Diagnostic performance of serological tests to detect antibodies against acute scrub typhus infection in central India. Indian J Med Microbiol. 2018;36(1):108-12.

9. Rajan SJ, Sathyendra S, Mathuram AJ. Scrub typhus in pregnancy: Maternal and fetal outcomes. Obstet Med. 2016;9(4):164-6.

10. Kumar R, Thakur S, Bhawani R, Kanga A, Ranjan A. Clinical Profile of Scrub Typhus in Pregnancy in Sub-Himalayan Region. J Obstet Gynecol India. 2016;66:82-7.

11. Suntharasaj T, Janjindamai W, Krisanapan S. Pregnancy with scrub typhus and vertical transmission: a case report. J Obstet Gynaecol Res. 1997 Feb;23(1):75-8.

12. Wang W, Sung N, Gilman-Sachs A, Kwak-Kim J. T Helper (Th) Cell Profiles in Pregnancy and Recurrent Pregnancy Losses: Th1/Th2/Th9/Th17/Th22/Tfh Cells. Front Immunol. 2020;11(August):1-14.

13. Sharma K, Sharma S, Chander D. Evaluation of Audio-Rhinological Changes During Pregnancy. Indian J Otolaryngol Head Neck Surg. 2011;63(1):74-8.

\section{Hosted file}

images scrubtyphus.pdf available at https://authorea.com/users/398684/articles/515443-scrubtyphus-in-pregnancy-presenting-with-permanent-hearing-loss-a-case-report 